\title{
Research on the GM(1,1) Method in Nuclear-Explosion-Yield Detection
}

\author{
Zhao Xiliang $^{1, a}$, Zhang Minghu ${ }^{2, b}$, Chen Hongmin ${ }^{3, b}$ and Mu Zhongguo ${ }^{2, a}$ \\ ${ }^{1}$ Dept. of Hydrographic and Mapping, Dalian Naval Academy, Dalian, China \\ ${ }^{2}$ Dept. of Underwater Weaponry and Chemical Defense, Dalian Naval Academy, Dalian, China \\ ${ }^{3}$ The Detachment of Warship Training, Dalian Naval Academy, Dalian, China \\ azmhly20040318@sina.com, ${ }^{b} z m h l y 20070618 @ 163 . c o m$
}

Keywords: Nuclear-explosion-yield Detection, GM(1,1) Modeling, Data Preparation, Parameters Estimation.

\begin{abstract}
For solving the questions that the practical value of the existing nuclear-explosion-yield detecting methods are excessively low, based on the $\mathrm{GM}(1,1)$ and the precondition of plenty nuclear explosion test data opened by home and overseas, the new model detecting nuclear explosion yield has been developed, and using the state estimating equation of the data precondition, a kind of new optimal estimation method detecting the nuclear explosion yield and a kind of means identifying the bad data have been established. The result of the validation applying nuclear explosion test data indicated that the new optimal estimation method using the grey theory to estimate the nuclear explosion yield is feasible. Compared with the existing detecting technology, the new estimation method can reduce error range, advance precision, offset the deficiencies of the existing means detecting the nuclear explosion yield, and enlarge the practical application value of the existing estimation models detecting the nuclear explosion yield.
\end{abstract}

\section{Introduction}

Nuclear explosion yield is the most important one of the nuclear explosion parameters, and it is the analysis basis of other nuclear explosion parameters. The grey theory has been widely applied in the military field, economics and sociology since it was developed in 1980s. The theory belongs to the systematization. The grey indicates the imperfection communication and the grey system is the system including imperfection information. The grey system is of the good characteristic, such as using a little information reach the high precision in establishing model. When the survey data of the nuclear explosion yield detecting are processed, some information can be obtained meanwhile others information can not be known, so the survey data processing system of the nuclear explosion yield detecting have the characteristics of the grey system, it can be analyzed by $\operatorname{GM}(1,1)$.

\section{Summarizing the methods of the existing nuclear explosion yield detecting}

There are the research methods about the existing nuclear explosion yield detecting, such as based the minimum illumination appearance time, based the mushroom cloud height after explosion, and based the steady-going parameters of the mushroom cloud. Reference [2] has a detailed presentation for the methods of the detecting nuclear explosion yield at home and abroad.

The existing nuclear explosion yield detecting means have disadvantages.

Using the minimum illumination appearance time to detect the yield. Because the minimum illumination appearance time is a millisecond-level datum, it is not feasible to detect the datum artificially, just being obtained using the expensively auto-observation instrument to survey. But it is very difficult to equip this kind of automatic observation instrument in mass instances, even though it has been equipped, because the scaled height correction factor is decided by height of explosion and the yield needed to determine, its complexity is obvious. Based on the above analysis, it can be found that the practical application value of this method is limited. 
Using mushroom cloud height after explosion in different time to detect the yield. The question of this method consists in whether the nuclear explosion moment time can reach the second-level exactitude or not. Otherwise, it is impossible to obtain the time after explosion, and to determine the yield. Especially, the method demands that the formula is chosen according to the guesstimate value of the yield unknown. So the practical application value of this method is very limited in wartime, too.

Using steady-going parameter of the mushroom cloud to detect the yield. The main question of this method is that it is difficult to determine the stabilization time of mushroom cloud, so that the detecting precision of the steady-going parameter of the mushroom cloud is lower. Meanwhile, the long time spent to stability mushroom cloud can not reach the demand of fast feedback in nuclear defense. Especially, the weather condition plays an important role in measuring steady-going parameter of the mushroom cloud, and sometimes it is impossible to detect these parameters. The steady time depends on the nuclear explosion moment time. So the practical application value of this method is also wondrously limited.

\section{Modeling of the grey theory}

The modeling of grey theory usually includes the modeling of the equal time list and the model of the non-equal time list. The time is mostly regarded as independent variable in processes of detecting nuclear explosion yield, so it is easy to arrive at the demand building equal time list model. Using the equal time list modeling, the pretreatment of nuclear explosion yield detecting data can enormously improve the practical application value of the existing detection methods. The main models of grey theory are as follows:

General Model of GM(1,1). Supposing the original list is as follows:

$$
X^{(0)}=\left\{x^{(0)}(1), x^{(0)}(2), \ldots, x^{(0)}(n)\right\} .
$$

The above list is a set of grey information which is of the characteristic of randomicity. It can be processed to offer the more useful information. This paper chooses a method of accumulate addition. The result is as follows:

$$
x^{(m)}(k)=\sum_{i=1}^{k} x^{(m-1)}(k) \quad k=1,2, \ldots, n .
$$

Usually the data processed with one time accumulate addition can take on the definite rules, and the times can be added if the rules are not visible.

Based on the processed data, the linear dynamic model is used to imitate and approach these data. The formula is as follows:

$$
\frac{d x^{(m)}}{d t}+a x^{(m)}=b
$$

The answers of the differential equations are:

$$
x^{(m)}(t+1)=\left[x^{(m-1)}(1)-\frac{b}{a}\right] e^{-a t}+\frac{b}{a} .
$$

The parameter of the differential equations can be obtained using least squares method, its vectors forms are:

$$
\hat{a}=\left[\begin{array}{ll}
a & b
\end{array}\right]^{T}=\left(B^{T} B\right)^{-1} B^{T} Y_{N} .
$$

In the formula:

$$
B=\left|\begin{array}{cc}
-0.5\left[x^{(1)}(1)+x^{(1)}(2)\right] & 1 \\
-0.5\left[x^{(1)}(2)+x^{(1)}(3)\right] & 1 \\
\ldots & \ldots \\
-0.5\left[x^{(1)}(n-1)+x^{(1)}(n)\right] & 1
\end{array}\right|, \quad Y_{N}=\left[\begin{array}{llll}
x^{(m-1)}(2) & x^{(m-1)}(3) & \ldots & \left.x^{(m-1)}(n)\right]^{T}
\end{array}\right.
$$


According as $\hat{x}^{(m-1)}(t+1)=\hat{x}^{(m)}(t+1)-\hat{x}^{(m)}(t)$ to accumulate subtracts, the data foreseen can be obtained.

If the model precision unfit for demand, residual error list can be built according as residual error accounted, and using residual error model to correct original model, lastly, the foresee equation can be obtained.

New Information Model of GM(1,1). Generally speaking, with changing of the object researched, the characteristic of new information to the system researched can obtain the more value than the old information. Based on this idea, the data obtaining new information are added to the original list to build GM(1,1) model, the kind of model built is called new information model of $\operatorname{GM}(1,1)$.

Equal Dimension Grey Data Substitute Model of GM(1,1). Using the model of GM(1,1) built with a list known forecast a datum, then bring the datum into the known list to substitute a old datum. The model of $\operatorname{GM}(1,1)$ is built with the above method, and the model just is equal dimension grey data substitute model of GM(1,1).

New Equal Dimension Model of GM(1,1). The model is found with the resembling method building new $\operatorname{GM}(1,1)$, the only difference is that using a new datum to replace a old datum.

\section{The pretreatment of the nuclear explosion yield data detected}

The Detecting Model of Nuclear Explosion Yield. Currently, the detecting technology of nuclear explosion yield in the world includes two aspects:

On the one hand, the nuclear explosion yield is detected with the vision parameters of the nuclear explosion appearance; On the other hand, the nuclear explosion yield is detected with the signal parameters of the nuclear explosion. So the detecting is indirect survey. The vision parameters of the nuclear explosion appearance or the signal parameters $\left(x_{1}, x_{2}, x_{3}, \ldots, x_{n}\right)$ are related with the nuclear explosion yield $Q$, and the function is as follows:

$$
Q=f\left(x_{1}, x_{2}, x_{3}, \ldots, x_{n}\right) \text {. }
$$

Considering some basic demands such as time, nicety and reliability, and some errors demands in the nuclear explosion yield detecting, and the survey errors of the nuclear explosion yield come from the detecting error of the vision or signal parameters, so when $\Delta x_{1}, \Delta x_{2}, \Delta x_{3}, \ldots, \Delta x_{n}$ are little, the yield errors can be confirmed with whole differential equation of (6) formula, the result is:

$$
\Delta Q=\frac{\partial f}{\partial x_{1}} \Delta x_{1}+\frac{\partial f}{\partial x_{2}} \Delta x_{2}+\frac{\partial f}{\partial x_{3}} \Delta x_{3}+\cdots \cdot \frac{\partial f}{\partial x_{n}} \Delta x_{n} .
$$

The above formula indicates that $Q$ should be a one dimension function of the vision or signal parameters and these parameters should be unattached random variable for reducing the yield detecting error and complexity.

The Pretreatment of Nuclear Explosion Yield Detecting Data. Usually, the data detected by survey instrument include some abnormal elements. Author regards these vision or signal parameters detected in the nuclear explosion as states, and takes the data pretreatment technology including the states estimation and the bad data identification into the grey system model. The general modeling is compared with the grey system modeling. The methods have greatly superiority at the aspect of improving precision of the grey system modeling.

\section{Analysis on the testing result of the nuclear explosion test data}

Based on these nuclear explosion data published by the nuclear-powered in the world, the data pretreatment is processed, then, the non-equal time list are converted into the equal time list, finally, the nuclear explosion yield detecting model is developed using the grey system theory.

Author chooses the nuclear explosion tests including four kinds of different yields from five nuclear countries, then, chooses five kinds of the representative data from every nuclear explosion 
testing data as original list, using the general model of $\operatorname{GM}(1,1)$, the new information model of $\operatorname{GM}(1,1)$, the equal dimension grey data substitute model of $\mathrm{GM}(1,1)$, and the new equal dimension model of $\operatorname{GM}(1,1)$ to process modeling estimation. The estimation results indicate that it is feasible to use the grey system theory to carry on the optimal estimation of the detecting value of nuclear explosion yield. Compared with the existing detecting technology, the new estimation method could reduce error range, advance precision. By the way, the precision of the estimation result of the new information model of $\mathrm{GM}(1,1)$ is the best, and the result of the general model of $\mathrm{GM}(1,1)$ is the worst.

\section{Conclusion}

Researches indicate: using the grey system theory and the method of the data pretreatment to estimate the detecting value of nuclear explosion yield, the model can be built by a few data, and it is possible to improve the precision of the model. The new modeling methods overcome the deficiencies of the existing means detecting nuclear explosion yield, and enlarge the practical application value of them. Meanwhile there are great application and reference value in the research of the engineering and sociology.

\section{Acknowledgements}

This work was financially supported in part by the national defense Basic Research Foundation of China (435B956).

\section{References}

[1] X. Zhang, M. Zhang. Nuclear Weapons and Warship Nuclear Defense, the CPLA Press, 2003.

[2] L. Jin, X. Wang, M. Zhang, etc. Chinese Journal of high pressure Physics, No.3 (2003), p. 230.

[3] DoD in US. NBC Defense Report, Washington D C: DoD in US, 1999.

[4] Y. Yan, M. Zhang, J. Wang. Rock and Soil Mechanics, No.5 (2006), p. 799.

[5] Y. Luo. Machine Tool \& Hydraulics, No. 6 (2005), p. 148.

[6] H. Zhang, W. Dai. Journal of Zhejiang Sci-Tech University, No. 1(2009), p. 142.

[7] B. Zeng, W. Zeng, Y. Luo. Journal of Liaoning Technical University, No. 5(2010), p. 934.

[8] W. Li. Computer Engineering and Applications, No. 14(2011), p. 25.

[9] X. He. Operations Research and Management Science, No. 6(2012), p. 23.

[10]K. Wang, Sh. Zhou. Science of Surveying and Mapping, No. 12(2014), p. 29.

[11] J. Wang. Mathematics in Practice and Theory, No. 12(2013), p. 111.

[12] L. Zeng, Sh. Luo. Mathematics in Practice and Theory, No. 1(2015), p. 88. 